International Journal of Pure and Applied Mathematics

Volume 85 No. 4 2013, 771-779

ISSN: 1311-8080 (printed version); ISSN: 1314-3395 (on-line version)

url: http://www.ijpam.eu

doi: http://dx.doi.org/10.12732/ijpam.v85i4.13

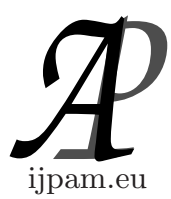

\title{
COMMON FIXED POINT FOR THREE NONSELF-MAPS THROUGH AN IMPLICIT RELATION
}

\author{
T. Phaneendra ${ }^{1}$, D. Surekha ${ }^{2}$, K. Kumara Swamy ${ }^{3}$ \\ ${ }^{1}$ Applied Analysis Division \\ School of Advanced Sciences \\ VIT University \\ Vellore, 632014, Tamil Nadu, INDIA \\ ${ }^{2}$ Department of Mathematics \\ Hyderabad Institute of Technology \& Management \\ RR District, Hyderabad (A.P.), INDIA \\ ${ }^{3}$ Department of Mathematics \\ Malla Reddy Engg. College for Women \\ Secunderabad, 500014 (A.P.), INDIA
}

\begin{abstract}
A common fixed point is obtained for three weakly compatible nonself-maps defined on a nonempty subset of a metric space through an implicit relation and the notion of property E.A. The result of the paper is a generalization of those of Sing and Kumar, and of Akkouchi and Popa, and is an extended generalization of Khan and Dolmo's result for a pair of maps.
\end{abstract}

AMS Subject Classification: $54 \mathrm{H} 25$

Key Words: property E.A. weakly compatible maps, implicit relation, common fixed point

\section{Introduction}

Let $(X, d)$ be a metric space. Then $S x$ denotes the image of $x \in X$ under a selfmap $S$ on $X$ and $S A$, the composition of self-maps $S$ and $A$ on $X$. Self-maps

Received: March 15, 2013

(C) 2013 Academic Publications, Ltd.

$\S$ Correspondence author url: www.acadpubl.eu 
$S$ and $A$ on $X$ are compatible [2] if

$$
\lim _{n \rightarrow \infty} d\left(S A x_{n}, A S x_{n}\right)=0
$$

whenever $\left\langle x_{n}\right\rangle_{n=1}^{\infty} \subset X$ such that

$$
\lim _{n \rightarrow \infty} S x_{n}=\lim _{n \rightarrow \infty} A x_{n}=p \quad \text { for some } \quad p \in X
$$

Therefore self-maps $S$ and $A$ on $X$ are noncompatible if (1.2) holds good but $\lim _{n \rightarrow \infty} d\left(S x_{n}, A x_{n}\right)$ is nonzero or $+\infty$ for at least one $\left\langle x_{n}\right\rangle_{n=1}^{\infty} \subset X$. It can be easily seen that both compatible and noncompatible maps are included in the class of maps with the choice (1.2). Self-maps $S$ and $A$ on $X$ are said to satify the property E.A. [1] if (1.2) holds good for some $\left\langle x_{n}\right\rangle_{n=1}^{\infty} \in X$. On the other hand, if $x_{n}=x$ for all $n$, the compatibility implies that $S A x=A S x$ whenever $A x=S x$. That is, $S$ and $A$ commute at their coincidence points. Self-maps which commute at their coincidence points are called weakly compatible [3]. However weak compatibility and property E. A. are independent notions of each other [6].

We aim at a common fixed point for three nonself-maps defined on a nonempty subset of a metric space through an implicit relation and the notions of weak compatibility and property E.A. Our contribution is a generalization of those of Singh and Kumar [7], and of Akkouchi and Popa [5]. This is also extended generalization of that of Khan and Dolmo [4], which was given for a pair of maps.

\section{Notation and Main Results}

In this paper, $X$ denotes a metric space, $Y$ an arbitrary non empty subset of $X$ and $G: \mathbb{R}_{+}^{6} \rightarrow \mathbb{R}$, a continuous function such that

$\left(G_{1}\right) G(u, 0,0, u, u, 0)>0$ for all $u>0$,

$\left(G_{2}\right) G(u, u, 0,0, u, u) \geq 0$ for all $u>0$,

$\left(G_{3}\right) G(u, 0, u, 0,0, u)>0$ for all $u>0$.

We prove 
Theorem 2.1. Let $S, T$ and $A: Y \rightarrow X$ satisfying the implicit contractivetype condition

$$
\begin{gathered}
G(d(S x, T y), d(A x, A y), d(A x, S x), d(A y, T y), d(A x, T y), d(A y, S x))<0 \\
\text { for all } \quad x, y \in Y,
\end{gathered}
$$

Suppose that one of the pairs $(S, A)$ and $(T, A)$ satisfies property E.A. on $Y$, and any one of the following conditions holds good:

1. $A(Y)$ is closed subspace of $Y$,

2. $\overline{S(Y)} \subset A(Y)$,

3. $\overline{T(Y)} \subset A(Y)$.

Then there is a coincidence point $u$ common to $S, T$ and $A$ in $Y$. Further if the point of common coincidence of $S, T$ and $A$ with respect to $u$ lies in $Y$, it will be their unique common fixed point, provided either $(S, A)$ or $(T, A)$ is a weakly compatible pair.

Proof. First suppose that $(S, A)$ satisfies the property E.A. on $Y$. Then there exists a $\left\langle p_{n}\right\rangle_{n=1}^{\infty} \in Y$ with the choice (1.2). We claim that $q=\lim _{n \rightarrow \infty} T p_{n}=$ $p$. If possible suppose that $d(p, q)>0$. Then writing $x=y=p_{n}$ in (2.1), we find that

$$
\begin{aligned}
G\left(d\left(S p_{n}, T p_{n}\right), d\left(A p_{n}, A p_{n}\right), d\left(A p_{n}, S p_{n}\right), d\left(A p_{n}, T p_{n}\right), d\left(A p_{n}, T p_{n}\right),\right. \\
\left.d\left(A p_{n}, S p_{n}\right)\right)<0 .
\end{aligned}
$$

Applying the limit as $n \rightarrow \infty$ in this and using (1.2), we get

$$
G(d(p, q), 0,0, d(p, q), d(p, q), 0) \leq 0,
$$

which would be a contradiction to $\left(G_{1}\right)$. Thus $p=q$ and hence

$$
\lim _{n \rightarrow \infty} S p_{n}=\lim _{n \rightarrow \infty} T p_{n}=\lim _{n \rightarrow \infty} A p_{n}=p \quad \text { for some } \quad p \in Y .
$$

Now let $(T, A)$ satisfy the property E.A. on $Y$ so that

$$
\lim _{n \rightarrow \infty} T p_{n}=\lim _{n \rightarrow \infty} A p_{n}=p \quad \text { for some } \quad p \in X
$$

for some $\left\langle p_{n}\right\rangle{ }_{n=1}^{\infty} \in Y$. If $s=\lim _{n \rightarrow \infty} S p_{n} \neq p$, using again (2.1) and proceeding as above, we get contradiction to the choice $\left(G_{1}\right)$. This proves $(2.2)$. 
Case $(a)$ : Suppose that $A(Y)$ is closed subspace of $Y$. Then $\left\langle A p_{n}\right\rangle{ }_{n=1}^{\infty}$ converges in $A(Y)$. Hence $p \in A(Y)$ so that $p=A u$ for some $u \in Y$.

First we claim that $S u=A u$. If $d(p, S u)=d(A u, S u)>0$, from (2.1) with $x=u$ and $y=p_{n}$, we would get

$G\left(d\left(S u, T p_{n}\right), d\left(A u, A p_{n}\right), d(A u, S u), d\left(A p_{n}, T p_{n}\right), d\left(A u, T p_{n}\right), d\left(A p_{n}, S u\right)\right)<0$.

Applying the limit as $n \rightarrow \infty$ and using (2.2), this gives

$$
G(d(S u, A u), 0, d(A u, S u), 0,0, d(A u, S u)) \leq 0
$$

which is a contradiction to $\left(G_{3}\right)$. Thus $d(A u, S u)=0$ or $A u=S u=p$.

Again if $d(p, T u)>0$, from (2.1) with $x=u=y$ it follows that

$$
G(d(S u, T u), d(A u, A u), d(A u, S u), d(A u, T u), d(A u, S u), d(A u, T u))<0
$$

or $G(d(S u, T u), 0,0, d(A u, T u), 0, d(A u, T u))<0$ contradicting $\left(G_{1}\right)$. Thus $d(p, T u)=0$, that is $S u=T u=A u=p$.

Case (b): Now suppose that (2) holds good. Then $\left\langle S p_{n}\right\rangle_{n=1}^{\infty} \subset S(Y)$ and hence lies in $\overline{S(Y)}$ so that $p \in \overline{S(Y)} \subset A(Y)$ and $u$ is a common coincidence by Case $(a)$.

Case (c): Now suppose that (3) holds good. Then as above we get that $\left\langle T p_{n}\right\rangle{ }_{n=1}^{\infty}$ converges in $\overline{T(Y)}$ and hence $p \in \overline{S(Y)} \subset A(Y)$. It follows that $u$ is a common coincidence from again Case $(a)$.

Suppose that $p \in Y$. If $(S, A)$ is weakly compatible, we find that

$$
S S u=S A u=A S u=A A u \text { or } A p=S p .
$$

If $S p \neq T p$ so that $d(S p, T p)>0$, then from (2.1), we get

$$
G(d(S p, T p), d(A p, A p), d(A p, S p), d(A p, T p), d(A p, T p), d(A p, S p))<0
$$

or $G(d(S p, T p), 0,0, d(S p, T p), d(S p, T p), 0)<0$, which is a contradiction to $\left(G_{1}\right)$. Thus $d(T p, S p)=0$ or $S p=A p=T p$.

On the other hand, if $(T, A)$ is weakly compatible, we find that

$$
T T u=T A u=A T u=A A u \text { or } A p=T p .
$$


Then (2.1) would give

$$
G(d(S p, T p), d(A p, A p), d(A p, S p), d(A p, T p), d(A p, T p), d(A p, S p))<0
$$

or $G(d(S p, T p), 0, d(T p, S p), 0,0, d(T p, S p))<0$, a contradiction to $\left(G_{3}\right)$ whenever $d(S p, T p)>0$. Thus $d(T p, S p)=0$ or $S p=A p=T p$.

Thus $p$ is also a common coincidence point of $S, T$ and $A$ in $Y$ whenever either $(S, A)$ or $(T, A)$ is weakly compatible.

Now if $p \neq S p$ so that $d(p, S p)=d(S u, T p)>0$ and from (2.1), we get

$$
G(d(S u, T p), d(A u, A p), d(A u, S u), d(A p, T p), d(A u, T p), d(A p, S u))<0
$$

or $G(d(p, S p), d(p, S p), 0,0, d(p, S p), d(S p, p))<0$ which is a contradiction to $\left(G_{2}\right)$. Thus $d(p, S p)=0$ and hence $p$ is a common fixed point of $S, T$ and $A$ in $Y$.

The uniqueness of the common fixed point can be easily obtained from (2.1) and the choice of $G$.

We write $G\left(t_{1}, t_{2}, t_{3}, t_{4}, t_{5}, t_{6}\right)=t_{1}-\max \left\{t_{2}, \alpha t_{3}, \alpha t_{4}, \frac{t_{5}+t_{6}}{2}\right\}$ in Theorem 2.1, where $0<\alpha<1$. Then

$\left(G_{1}\right) G(u, 0,0, u, u, 0)=u-\max \left\{0,0 . \alpha, \alpha \cdot u, \frac{u+0}{2}\right\}=(1-k) u>0$ for all $u>0$ where $k=\max \{\alpha, 1 / 2\}$,

$\left(G_{2}\right) G(u, u, 0,0, u, u)=u-\max \left\{u, \alpha .0, \alpha .0, \frac{u+u}{2}\right\}=u-u>0$ for all $u>0$,

$\left(G_{3}\right) G(u, 0, u, 0,0, u)=u-\max \left\{0, \alpha \cdot u, \alpha \cdot 0, \frac{0+u}{2}\right\}=(1-\alpha) u>0$ for all $u>0$.

Therefore we have

Corollary 2.1 (Theorem 2, [7]). Let $S, T$ and $A: Y \rightarrow X$ satisfy the inequality

$$
\begin{gathered}
d(S x, T y)<\max \left\{d(A x, A y), \alpha d(A x, S x), \alpha d(A y, T y), \frac{d(A x, T y)+d(S x, A y)}{2}\right\} \\
\text { for all } \quad x, y \in X
\end{gathered}
$$

where $0 \leq \alpha<1$. Suppose that both (2) and (3) of Theorem 2.1 hold good and one of the pairs $(S, A)$ and $(T, A)$ satisfies the property E.A. on $Y$. Then there is a coincidence point common to $S, T$ and $A$ in $Y$. Further if $Y=X$ and $(S, A)$ and $(T, A)$ are weakly compatible, then $S, T$ and $A$ will have a unique common fixed point in $X$. 
Remark 2.1. Corollary 2.1 required both the inclusions (2) and (3); weak compatibility of both the pairs $(S, A)$ and $(T, A)$. Also a common fixed point was obtained under the condition that $Y=X$. Our proof suggests that weak compatibility and property E.A. of either pair is sufficient to obtain a common fixed point, even without the condition that $Y=X$.

In extending the property E.A. to more than two self-maps, Akkouchi and Popa [5] defined a class $C$ of self-maps which satisfy property E.A. if there is a $\left\langle x_{n}\right\rangle{ }_{n=1}^{\infty} \subset X$ such that $\lim _{n \rightarrow \infty} S x_{n}=p$ for each $S \in C$ for some $p \in X$.

Then the following was proved:

Theorem 2.2 (Theorem 2, [5]). Let $S, T$ and $A$ be self maps on $X$ satisfying the inclusions

$$
S(X) \subset A(X) \text { and } T(X) \subset A(X)
$$

and the implicit condition (2.1) with $x \neq y$. Suppose that the triplet $(S, T, A)$ satisfies property E.A. and any one of $A(X), S(X)$ and $T(X)$ is a closed subspace of $X$. If $(S, A)$ and $(T, A)$ are weakly compatible, then $S, T$ and $A$ will have a unique common fixed point.

We claim that Theorem 2.2 is a special case of Theorem 2.1 with the help of the following

Lemma 2.1. The following three conditions are equivalent under (2.1)

(a) $(S, A)$ satisfies property E.A.

(b) $(T, A)$ satisfies property E.A.

(c) the triad $(S, T, A)$ satisfies property E.A.

Proof. We establish the equivalence through arguments:

$(c) \Rightarrow(a),(c) \Rightarrow(b),(a) \Rightarrow(b) \Rightarrow(c)$ and $(b) \Rightarrow(a) \Rightarrow(c)$.

Note that $(c) \Rightarrow(a)$ and $(c) \Rightarrow(b)$ are obvious.

$(a) \Rightarrow(b) \Rightarrow(c)$ : Suppose that (a) holds good. Then there is a $\left\langle x_{n}\right\rangle \underset{n=1}{\infty} \in X$ with the choice (1.2). We claim that $q=\lim _{n \rightarrow \infty} T x_{n}=p$. If possible suppose that $d(p, q)>0$. Then writing $x=y=x_{n}$ in (2.1), we see that

$$
\begin{aligned}
G\left(d\left(S x_{n}, T x_{n}\right), d\left(A x_{n}, A x_{n}\right), d\left(A x_{n}, S x_{n}\right), d\left(A x_{n}, T x_{n}\right), d\left(A x_{n}, T x_{n}\right),\right. & \\
\left.d\left(A x_{n}, S x_{n}\right)\right) & <0 .
\end{aligned}
$$

Applying the limit as $n \rightarrow \infty$ in this and using (1.2), we get a contradiction to $\left(G_{1}\right)$ that $G(d(p, q), 0,0, d(p, q), d(p, q), 0) \leq 0$. Thus $p=q$ and (b) and hence (c) holds good. 
$(b) \Rightarrow(a) \Rightarrow(c):$ If $(\mathrm{b})$ holds good, then

$$
\lim _{n \rightarrow \infty} T y_{n}=\lim _{n \rightarrow \infty} A y_{n}=r \quad \text { for some } \quad r \in X
$$

for some $\left\langle y_{n}\right\rangle{ }_{n=1}^{\infty} \in X$. Let $d(r, s)>0$ where $s=\lim _{n \rightarrow \infty} S y_{n}$. Then (2.1) with $x=y=y_{n}$ gives

$$
\begin{array}{r}
G\left(d\left(S y_{n}, T y_{n}\right), d\left(A y_{n}, A y_{n}\right), d\left(A y_{n}, S y_{n}\right), d\left(A y_{n}, T y_{n}\right), d\left(A y_{n}, T y_{n}\right),\right. \\
\left.d\left(A y_{n}, S y_{n}\right)\right)<0 .
\end{array}
$$

Applying the limit as $n \rightarrow \infty$ in this and using (2.5), we get

$$
F(d(s, r), 0, d(r, s), 0,0, d(r, s)) \leq 0,
$$

which is also a contradiction to $\left(G_{2}\right)$. Thus $s=r$ so that (a) and hence (c) holds good.

The following example shows that (a) and (b) need not imply (c) without (2.1):

Example 2.1. Let $X=\mathbb{R}_{+}$with the usual metric $d$. Define $S, A: X \rightarrow X$ by $S x=a x+b, T x=(b-a) x+a$ and $A x=c x+b$ for all $x \in X$, where $a, b$ and $c$ are nonnegative numbers with $a \neq c$ and $b-a-c>0$. Choose $x_{n}=\frac{1}{n} \quad$ and $\quad y_{n}=\frac{b-a}{b-a-c}+\frac{1}{n} \quad$ for all $n$ so that

$$
\lim _{n \rightarrow \infty} S x_{n}=b=\lim _{n \rightarrow \infty} A x_{n} \text { and } \lim _{n \rightarrow \infty} T y_{n}=\lim _{n \rightarrow \infty} A y_{n}=\frac{b^{2}-a b-a c}{b-a-c} .
$$

That is, $(S, A)$ and $(T, A)$ satisfy the property E.A. By a routine computation, it can be easily seen that $(S, T, A)$ does not satisfy the property E.A., though the individual pairs satisfy the property E. A.

Now take $a=\frac{1}{2}, b=1, c=\frac{1}{2}$ and

$$
G\left(t_{1}, t_{2}, t_{3}, t_{4}, t_{5}, t_{6}\right)=t_{1}-q \max \left\{t_{2}, t_{3}, t_{4}, \frac{t_{5}+t_{6}}{2}\right\},
$$

where $0 \leq q<1$. Then (2.1) becomes

$$
\begin{gathered}
d(S x, T y) \leq q \max \left\{d(A x, A y), d(A x, S x), d(A y, T y), \frac{d(T y, A x)+d(S x, A y)}{2}\right\} \\
\text { for all } \quad x, y \in X \quad \text { with } \quad x \neq y
\end{gathered}
$$

we see that for $x=2$ and $y=0$, we see that its LHS is $\frac{3}{2}$ while RHS is $\frac{13}{12}$ so that (2.6) holds good only if $q \geq \frac{18}{13}$ which is against the choice of $q$. Thus (2.6) fails. 
Remark 2.2. In view of Lemma 2.1, Theorem 2.2 follows as a particular case of our result when $Y=X$. Here we note that Theorem 2.2 required weak compatibility of both the pairs $(S, A)$ and $(T, A)$. If $S(X)$ is closed in $X$, then hence $\overline{S(X)}=S(X) \subset A(X)$, due to (2.4). Similarly if $T(X)$ is closed so that $\overline{T(X)}=T(X) \subset A(X)$, again from (2.4). It is thus interesting to note that the completeness of $A(X)$ is weakened and the inclusions (2.4) and the completeness of $S(X)$ and $T(X)$ are chipped in (2) and (3).

No we set $F\left(t_{1}, t_{2}, t_{3}, t_{4}, t_{5}, t_{6}\right)=t_{1}-\max \left\{t_{2}, \beta t_{3}+\alpha t_{4}, \frac{t_{5}+t_{6}}{2}\right\}$, where $\beta \geq 0$ and $0<\alpha<1$. Then

$\left(F_{1}\right) F(u, 0,0, u, u, 0)=u-\max \left\{0,0 . \beta+\alpha . u, \frac{u+0}{2}\right\}=(1-k) u>0$ for all $u>0$ where $k=\max \{\alpha, 1 / 2\}<1$,

$\left(F_{2}\right) F(u, u, 0,0, u, u)=u-\max \left\{u, 0 . \beta+\alpha \cdot 0 \frac{u+u}{2}\right\}=u-u>0$ for all $u>0$.

With this choice and $T=S$ in Theorem 2.1, we get the following result due to Khan and Dolmo [4]:

Corollary 2.2. Let $S, A: Y \rightarrow X$ satisfying

$$
\begin{gathered}
d(S x, S y)<\max \left\{d(A x, A y), \beta d(S x, A x)+\alpha d(S y, A y), \frac{d(S y, A x)+d(S x, A y)}{2}\right\} \\
\text { for all } x, y \in Y \quad \text { with } \quad x \neq y,
\end{gathered}
$$

where $\beta \geq 0$ and $0<\alpha<1$. Suppose that either $A(Y)$ is a complete subspace of $Y$ or $S(Y)$ is a complete subspace of $Y$ with $S(Y) \subset A(Y)$. Then $(S, A)$ have a coincidence point a in $Y$. Further if the point of coincidence of $S$ and $A$ with respect to a is in $Y$, then $S$ and $A$ will have a unique unique common fixed point in $Y$, provided $S$ and $A$ are weakly compatible.

\section{Acknowledgments}

The authors are highly thankful to the referees for their valuable suggestions in improving the paper.

\section{References}

[1] Aamri, M.A. and Moutawakil, D. El., Some new common fixed point theorems under strict contractive conditions, J. Math. Anal. Appl., 270 (2002), $181-188$ 
[2] Gerald Jungck, Compatible maps and common fixed points, Int.J. Math. \& Math. Sci., 9 (1986), 771-779

[3] Gerald Jungck and Rhoades, B. E., Fixed point for set valued functions without continuity, Indian J. pure appl. Math., 29 (1998), 227-238

[4] Khan, A.R. and Domlo, A.A., Coincidence and fixed points of nonself contractive maps with applications, Ind. Jour. Math. 49 (2007), 17-13

[5] Mohammad Akkouchi and Valeriu Popa, Well-posedness of a common fixed point problem for three mappings under strict contractive conditions, Buletin. Univers. Petrol-Gaze din Ploiesti, Seria Math. Inform. Fiz., 61 (2009), 1-10

[6] Pathak, H. K., Cho, Y. J. and Kang, S. M., Remarks on R-weakly commuting mappings and common fixed point theorems, Bull. Korean Math. Soc., 17 (1997), 247-257

[7] Singh, S. L. and Ashish Kumar, Common fixed point theorems for contractive maps, Math. Vesnik. 58 (2006), 85-90. 
